\title{
A contribution to the hypothesis of nicotinic challenge as therapeutic option for COVID-19 patients
}

\author{
Luigi Manni', Paolo Tieri ${ }^{1}$, Marzia Soligo ${ }^{1}$
}

1 Italian National Res earch Council

\begin{abstract}
The pandemic caused by SARS-CoV-2 represents an open and unresolved challenge for the global health system. The need to identify drugs that demonstrate efficacy in countering both the mechanisms of interaction of SARS-CoV-2 with host cells and to control the devastating inflammatory phenomena that characterize the late stages of viral infection, requires increasingly urgent answers. The biomedical research approach based on the repurposing of already approved drugs seems to be one of the most viable strategies in this struggle. In this work, by using a computational pharmacology approach and on the basis of what has been recently reported on the potential of nicotinic receptors in countering both phases of COVID-19, we propose a hypothesis aimed at widening the spectrum of pharmacological tools currently available to medical doctors. Our proposal specifically concerns the possibility of using tropisetron, a 5-HT3 receptor antagonist at the same time positive allosteric interactor of the nicotinic alpha-7 receptor, in order to inhibit unexplored virus-host interaction and restore the physiological control mechanisms of the excessive inflammation caused by SARS-CoV-2.
\end{abstract}

In the context of the frantic search for therapeutics useful to tackle the current pandemic of SARS-CoV-2 and to limit the enormous burden on the intensive care units and health systems of the countries involved, relevant emphasis has been put on strategies aimed at repurposing drugs already approved for other conditions. An original and, in our opinion, remarkable contribution has been recently put forward, based on clinical epidemiological analyses (Miyara et al., 2020) and on the current knowledge of neuroimmune modulation of the inflammatory response (Changeux et al., 2020), proposing the use of nicotine to interfere with unexplored virus-host interactions and to face the tremendous impact of the cytokine storm syndrome characterizing the second phase of COVID-19. Here, we would like to extend and integrate such hypothesis and 
suggest further therapeutic options based on the challenge of alpha-7 nicotinic acetylcholine receptor ( $\alpha 7 \mathrm{nAchR}$, gene name: CHRNA7).

We are currently finalizing an extensive investigation of SARS-CoV-2 host interactome, performed via a network medicine approach (Silverman et al., 2020) based on available virus-host protein-protein interaction (PPI) data, and on integ rated computational analyses (including machine learning), aimed to identify a limited host gene set as possible drug targets (the methodological details of such analysis will be illustrated and extensively discussed elsewhere (T ieri P. et al, manuscript in preparation)). In our preliminary computational analysis of the SARS-CoV-2 host interactome we identified genes with high centrality (betweenness centrality rank $<50$ ) and a strong connection with some peculiar symptoms of COVID-19 patients, such as anosmia and ageusia, among others (VarElect suite, (Stelzer et al., 2016)) (preliminary unpublished data at https://www.iac.cnr.it/ tieri/projects/COVID-19/covid-19.html), that may be potential pharmacological targets for the treatment of COVID-19. Among high scoring genes, our attention was attracted in particular, as neuropharmacolog ists, by the HTR3A gene, whose protein is a direct interactor of FURIN (Huttlin et al., 2017), this latter proposed as a critical and specific protease for Sars-CoV-2 Spike (S) protein cleavage, and subsequently suggested as a potential drug target (Jaimes et al., 2020; Mallapaty, 2020; Wu et al., 2020). HTR3A gene codifies for the A subunit of the serotonin ionotropic 5HT3 receptor, that is a neurotransmitter receptor belonging to the Cys-loop superfamily of ligand-gated ion channels (CL-LGICs), expressed in the central and peripheral nervous system with relevant pharmacological value in psychiatric and gastrointestinal diseases (Juza et al., 2020). Searching for specific correlates of 5-HT3 dysfunction and SARS-CoV2 infection, we noticed that this serotonin receptor is important, among others, in the signaling between taste buds and gustatory nerves, accounting for a significant proportion of the neural taste response (Larson et al., 2015). Thus a possible correlation between 5-HT3 and the taste dysfunction (ageusia/dysgeusia) reported as a peculiar symptom in a great percentage of COVID-19 patients (Lechien et al., 2020), is conceivable.

The hypothesis of a possible complex interplay among viral proteins, Furin and the 5-HT3 receptor, supported by previous (Huttlin et al., 2017; Canrong et al., 2020) and our ongoing computational analyses could account for its loss-of-function. It is well established that the receptor-binding domains on the SARS-CoV-2 S proteins bind with high affinity to human ACE2, an interaction accounting for virus entry in the host cell and for its transmissibility (Wrapp et al., 2020). However, the awareness that additional virushost interactions, not necessarily related with viral transmission of disease, could determine the insurgence and progression of symptoms is gaining ground. Zhou et al. 
noted unique features on a separate ( $\mathrm{N}$-terminal) domain of the SARS-CoV-2 $\mathrm{S}$ proteins that may confer binding to alternative host-cell receptors (Zhou et al., 2020) and it is known that analogous domains on several human CoVs have important auxiliary cellbinding functions (Park et al., 2019). Evidence for alternative interaction of virus S protein with receptors other than ACE2 have been also recently suggested by computational analysis (Milanetti et al., 2020). Notably, Changeux and colleagues also based their nicotinic hypothesis on the assumption of possible direct interaction of viral proteins with nAChRs (Chang eux et al., 2020).

We thus searched for other 5-HT3 structurally-related proteins, possibly correlating with one or more COVID-related symptoms and disease manifestations, in particular the uncontrolled and sustained inflammatory response, obviously mediated by over-activity of pro-inflammatory cytokines, as IL-1 beta, TNF-alpha or IL-6. This search attracted our attention to another neurotransmitter receptor, belong ing to the CL-LGICs superfamily, with relevant structural and pharmacological similarities to the 5-HT3 receptor, namely the $\alpha 7$ nAchR, (Zwart et al., 2004). Thus we may hypothesize that SARS-CoV-2 virus interacts with, and affects the function of LGICs, and that such interactions could account for the development of known COVID-19 clinical manifestations.

As put forward by Changeux and colleagues (Changeux et al., 2020), $\alpha 7$ nAchR presents some peculiar characteristics that make it a strong candidate as a therapeutic target in the COVID-19 pandemic. Besides its distribution and role in the central and peripheral nervous system, this receptor plays a crucial role in the homeostatic regulation of inflammatory processes. According to the inflammatory reflex theory (Tracey, 2002; Martelli, Farmer and Yao, 2016; Maturo et al., 2020), the $\alpha$ 7nAchR is at the interface between the immune and nervous systems, expressed on cytokine-producing monocytes and its activation by acetylcholine (ACh) dampens the inflammatory process by downregulating pro-inflammatory cytokines production. It is worth noting that the blockade of $\alpha 7 \mathrm{nAchR}$ functions by viral interactors may selectively worsen the SARS-CoV2-mediated uncontrolled inflammation in the lungs (Gahring et al., 2017).

Thus, we hypothesize that both 5-HT3 and $\alpha 7 \mathrm{nAchR}$, due to their structural and pharmacological similarities (Zwart et al., 2004), may represents possible alternative targets for virus-host interaction, with possible de-sensitization or de-activation of both receptors and the consequent development of at least lung inflammation and ageusia. Our working hypothesis, actually based on the preliminary bioinformatics analyses and pharmacological speculations, is thus to recover the conceivable $\alpha 7 \mathrm{nAchR}$ loss-offunction by a pharmacological approach based on its shared structural/pharmacological 
features with the 5-HT3 receptor (Zwart et al., 2004). The use of a positive allosteric modulator of $\alpha 7 \mathrm{nAchR}$, such as the 5-HT3 antagonist tropisetron, yet used for gastroenteric symptoms, is, in our opinion, a valuable choice, in order to firstly maintain a proper safety profile in patients yet challenged by a potentially lethal condition (Stegemann and Böhm, 2019). A similar approach has been proposed, suggesting the positive allosteric $\alpha 7$ nAchR modulator ivermectin (Changeux et al., 2020), which has been already reported as a possible inhibitor of SARS-CoV-2 replication (Caly et al., 2020). In our opinion, the possibility to target two possible virus-interacting host proteins by a single drug may represent a significant integrative advantage. Indeed, Tropisetron is both a selective serotonin receptor antagonist, which competitively blocks the action of serotonin at 5HT3 receptors ('T ropisetron', no date) and a positive allosteric modulator of the $\alpha 7 n A c h R$ (Khalifeh et al., 2015). Furthermore, the $\alpha 7 n A c h R$-based anti-inflammatory action of the drug has already been demonstrated in preclinical studies (T asaka et al., 2015; Stegemann and Böhm, 2019). It is thus conceivable that, by physical interacting with both receptors, tropisetron may positively interfere with some of the mechanisms of virus entry into host's cells, at the same time triggering the activation of physiological mechanisms controlling and damping excessive inflammation.

In conclusion, based on the cholinergic anti-inflammatory pathway as a potential target for drug repurposing in the fight against SARS-CoV-2 pandemics, we strongly support the hypothesis of nicotinic challenge as a proper pharmacological strategy to prevent or dampen the over-inflammation characterizing the second phase of viral infection. We propose a therapeutic strategy aimed at potentiating the activity of $\alpha 7 \mathrm{nAchR}$ and at interfering with possible CL-LGICs-viral direct interactions, based on the use of the antiemetic drug tropisetron. Such drug may probably be combined with nicotine, in a supportive interaction. The evidence that an approach based on the pharmacological enhancement of the endogenous ACh content by means of anti-cholinesterase, proposed for an ongoing patient-recruiting trial in Mexico (Pyridostigmine in Severe SARS-CoV-2 Infection - Full Text View - ClinicalT rials.gov, no date), strengthens such working hypothesis. Indeed, it suggests the possibility for a combination therapy based on Tropisetron and/or nicotine and/or Pyridostigmine, an acetylcholinesterase inhibitor, aimed at restoring the cholinergic anti-inflammatory pathway by increasing both the availability of the ligand (ACh or nicotine) and the responsivity of its specific $\alpha 7 \mathrm{nAch}$ receptor.

Acknowledgements

The authors thank Drs. Massimiliano Adamo, Filippo Castiglione, Christine Nardini, 
Daniele Santoni, Paola Stolfi, Davide Vergni for fruitful discussion, ideas and support in conceiving and elaborating this hypothesis.

\section{References}

Caly, L. et al. (2020) 'T he FDA-approved drug ivermectin inhibits the replication of SARSCoV-2 in vitro', Antiviral research, 178, p. 104787.

Canrong, W. U. et al. (2020) 'Furin, a potential therapeutic target for COVID-19', In: ChinArxiv [Internet]. Available at: http://www.chinaxiv.org/abs/202002.00062.

Changeux, J.-P. et al. (2020) 'A nicotinic hypothesis for Covid-19 with preventive and therapeutic implications', Qeios. doi: 10.32388/FXGQSB.2.

Gahring, L. C. et al. (2017) 'Nicotinic alpha 7 receptor expression and modulation of the lung epithelial response to lipopolysaccharide', PloS one, 12(4), p. e0175367.

Huttlin, E. L. et al. (2017) 'Architecture of the human interactome defines protein communities and disease networks', Nature, 545(7655), pp. 505-509.

Jaimes, J. A. et al. (2020) 'Structural modeling of 2019-novel coronavirus (nCoV) spike protein reveals a proteolytically-sensitive activation loop as a distinguishing feature compared to SARS-CoV and related SARS-like coronaviruses', Microbiology. bioRxiv https://doi.org/10.1101/2020.02.10.942185.

Juza, R. et al. (2020) 'Recent advances with 5-HT3 modulators for neuropsychiatric and gastrointestinal disorders', Medicinal research reviews. doi: 10.1002/med.21666.

Khalifeh, S. et al. (2015) 'Beyond the 5-HT3 receptors: A role for $\alpha$ 7nACh receptors in neuroprotective aspects of tropisetron', Human \& experimental toxicology, 34(9), pp. 922-931.

Larson, E. D. et al. (2015) 'The Role of 5-HT3 Receptors in Signaling from Taste Buds to Nerves', The Journal of neuroscience: the official journal of the Society for Neuroscience, 35(48), pp. 15984-15995.

Lechien, J. R. et al. (2020) ‘Olfactory and gustatory dysfunctions as a clinical presentation of mild-to-moderate forms of the coronavirus disease (COVID-19): a multicenter 
European study', European archives of oto-rhino-laryngology: official journal of the European Federation of Oto-Rhino-Laryngological Societies : affiliated with the German Society for Oto-Rhino-Laryngology - Head and Neck Surgery. doi: 10.1007/s00405-02005965-1.

Mallapaty, S. (2020) 'Why does the coronavirus spread so easily between people?', Nature, 579(7798), p. 183.

Martelli, D., Farmer, D. G. S. and Yao, S. T. (2016) ‘T he splanchnic anti-inflammatory pathway: could it be the efferent arm of the inflammatory reflex?', Experimental physiology, 101(10), pp. 1245-1252.

Maturo, M. G. et al. (2020) ‘The greater inflammatory pathway-high clinical potential by innovative predictive, preventive, and personalized medical approach', The EPMA journal, 11(1), pp. 1-16.

Milanetti, E. et al. (2020) 'In-Silico evidence for two receptors based strategy of SARSCoV-2', arXiv [physics.bio-ph]. Available at: http://arxiv.org/abs/2003.11107.

Miyara, M. et al. (2020) 'Low incidence of daily active tobacco smoking in patients with symptomatic COVID-19', Qeios. doi: 10.32388/WPP19W.3.

Park, Y.-J. et al. (2019) ‘Structures of MERS-CoV spike glycoprotein in complex with sialoside attachment receptors', Nature structural \& molecular biology, 26(12), pp. 11511157.

Pyridostigmine in Severe SARS-CoV-2 Infection - Full Text View - ClinicalT rials.gov (no date). Available at: https://www.clinicaltrials.gov/ct2/show/NCT 04343963 (Accessed: 24 April 2020).

Silverman, E. K. et al. (2020) 'Molecular networks in Network Medicine: Development and applications', Wiley interdisciplinary reviews. Systems biology and medicine, p. e1489.

Stegemann, A. and Böhm, M. (2019) ‘T ropisetron via $\alpha 7$ nicotinic acetylcholine receptor suppresses tumor necrosis factor- $\alpha$-mediated cell responses of human keratinocytes', Experimental dermatology, 28(3), pp. 276-282. 
Stelzer, G. et al. (2016) 'VarElect: the phenotype-based variation prioritizer of the GeneCards Suite', BMC genomics, 17 Suppl 2, p. 444.

Tasaka, Y. et al. (2015) 'Involvement of stimulation of $\alpha 7$ nicotinic acetylcholine receptors in the suppressive effect of tropisetron on dextran sulfate sodium-induced colitis in mice', Journal of pharmacological sciences, 127(3), pp. 275-283.

Tracey, K. J. (2002) 'T he inflammatory reflex', Nature, 420(6917), pp. 853-859.

'T ropisetron' (no date). Available at: https://www.drugbank.ca/drugs/DB11699 (Accessed: 24 April 2020).

Wrapp, D. et al. (2020) 'Cryo-EM structure of the 2019-nCoV spike in the prefusion conformation', Science, 367(6483), pp. 1260-1263.

Wu, C. et al. (2020) Furin, a potential therapeutic target for COVID-19, ChinArxiv. Available at: http://www.chinaxiv.org/abs/202002.00062 (Accessed: 24 April 2020).

Zhou, P. et al. (2020) 'A pneumonia outbreak associated with a new coronavirus of probable bat orig in', Nature, 579(7798), pp. 270-273.

Zwart, R. et al. (2004) 'Common structural and pharmacological properties of serotonin 5-HT3 receptors and_7 nicotinic acetylcholine receptors', in Cholinergic Mechanisms. unknown, pp. 309-315. 